А. В. Аргучинцев, В. С. Кедрин, М. С. Кедрина. Вариационное условие оптимальности в задаче управления гиперболическими уравнениями ...

Научная статья

УДК 517.977

doi: 10.18101/2304-5728-2021-1-13-23

\title{
ВАРИАЦИОННОЕ УСЛОВИЕ ОПТИМАЛЬНОСТИ В ЗАДАЧЕ УПРАВЛЕНИЯ ГИПЕРБОЛИЧЕСКИМИ УРАВНЕНИЯМИ С ДИНАМИЧЕСКИМИ ГРАНИЧНЫМИ УСЛОВИЯМИ
}

\section{(C) Аргучинцев Александр Валерьевич}

доктор физико-математических наук, профессор, Иркутский государственный университет Россия, 664003, г. Иркутск, ул. Карла Маркса, 1 arguch@math.isu.ru

\section{(C) Кедрин Виктор Сергеевич}

кандидат технических наук, доцент, Иркутский государственный университет Россия, 664003, г. Иркутск, ул. Карла Маркса, 1 kedrinvs@isu.ru

\section{(C) Кедрина Мария Сергеевна}

магистрант,

Иркутский государственный университет

Россия, 664003, г. Иркутск, ул. Карла Маркса, 1

kedrinams@gmail.com

\begin{abstract}
Аннотация. В статье рассматривается задача оптимального управления линейной системой гиперболических уравнений первого порядка с квадратичным целевым функционалом и граничными условиями, определяемыми из управляемых билинейных дифференциальных уравнений. Задачи такого типа возникают при моделировании ряда процессов химической технологии, социальной демографии и динамики популяций. В силу билинейности обыкновенных дифференциальных уравнений для решения подобных задач обычно применяют общие методы оптимального управления. Осуществлена редукция к задаче оптимального управления системой обыкновенных дифференциальных уравнений. Редукция основана на неклассических формулах приращения целевого функционала второго порядка. Такой подход позволяет применять ряд эффективных методов оптимального управления для решения исходной задачи.
\end{abstract}

Ключевые слова: гибридные системы; гиперболические уравнения; неклассические формулы приращения; редукция задач оптимального управления.

\section{Благодарности}

Исследование выполнено при финансовой поддержке РФФИ и Правительства Иркутской области в рамках научного проекта 20-41-385002, а также при поддержке РФФИ в рамках научного проекта 20-07-00407. 


\section{Для цитирования}

Аргучинцеев А. В., Кедрин В. С., Кедрина М. С. Вариационное условие оптимальности в задаче управления гиперболическими уравнениями с динамическими граничными условиями // Вестник Бурятского государственного университета. Математика, информатика. 2021. № 1. С. 13-23.

\section{Введение}

При моделировании процессов химической ректификации, социальной демографии, математической биологии в ряде случаев возникает необходимость решения задач оптимального управления гиперболическими системами первого порядка с начально-краевыми условиями, определяемыми из управляемых систем обыкновенных дифференциальных уравнений [1-3]. При этом обыкновенные дифференциальные уравнения иногда имеют специфическую структуру: фазовые переменные входят линейно в правые части динамических систем, но с зависящими от управлений коэффициентами. В силу этой билинейности принцип максимума Л. С. Понтрягина не является достаточным условием оптимальности даже в случае линейных гиперболических систем и линейного целевого функционала. Поэтому обычно для решения подобных задач применяют общие методы нелинейной теории оптимального управления.

В работе [4] данный тип задач был исследован для одного частного варианта гиперболических систем и линейного целевого функционала. В отличие от классического варианта формулы приращения, берущего начало еще от работы Л. И. Розоноэра [5], было получено два вида точных (без остаточных членов) формул приращения для произвольной пары допустимых процессов. Данный результат позволил провести редукцию исходной задачи оптимального управления к задаче оптимального управления обыкновенными дифференциальными уравнениями.

В настоящей работе рассматривается случай гиперболических систем, характерных для задач моделирования динамики популяций с учетом их возрастной структуры [6]. Целью управления является минимизация нормы конечного состояния. В силу квадратичности целевого функционала пришлось применить формулы приращения второго порядка, обычно используемые при исследовании особых оптимальных процессов [7]. Установлена справедливость необходимого и достаточного условия оптимальности вариационного типа. Изложена схема редукции исходной задачи к задаче оптимального управления системой обыкновенных дифференциальных уравнений, основанная на полученном результате.

\section{1 Постановка задачи}

Рассматривается задача оптимального управления системой линейных гиперболических уравнений первого порядка с линейной правой частью:

$$
\frac{\partial x}{\partial t}+\frac{\partial x}{\partial s}=B(t, s) x+r(t, s) .
$$


А. В. Аргучинцев, В. С. Кедрин, М. С. Кедрина. Вариационное условие оптимальности в задаче управления гиперболическими уравнениями ...

Здесь $x(t, s)-n$-мерная вектор-функция состояния процесса, $B(t, s)$ - матрица коэффициентов, независимые переменные $t$ и $s$ принимают значения из отрезков $T=\left[t_{0}, t_{1}\right]$ и $S=\left[s_{0}, s_{1}\right]$ соответственно. Начальные условия фиксированы:

$$
x\left(t_{0}, s\right)=x^{0}(s), s \in S .
$$

Предполагается, что граничные условия при $s=s_{0}$ определяются из управляемой системы обыкновенных дифференциальных уравнений:

$$
\frac{\partial x\left(t, s_{0}\right)}{\partial t}=C(u(t), t) x\left(t, s_{0}\right)+q(u(t), t) .
$$

В системе (3) $u(t)-m$-мерная вектор-функция управления из класса ограниченных и измеримых на отрезке $T$ функций. Начальные условия для (3) определяются из требования выполнения условия согласования с (2):

$$
x\left(t_{0}, s_{0}\right)=x^{0}\left(s_{0}\right) .
$$

Предполагаем, что управляющие функции почти всюду на отрезке $T$ удовлетворяют условию типа включения:

$$
u(t) \in U \subset E^{m} .
$$

Целью задачи оптимального управления является минимизация функционала:

$$
J(u)=\frac{1}{2} \int_{S}\left\|x\left(t_{1}, s\right)-z(s)\right\|^{2} d s .
$$

Здесь под нормой понимается обычная евклидова норма в $n$-мерном пространстве, $z(s)$ - заданная функция.

Задачи такого нестандартного типа возникают при моделировании ряда процессов химической технологии и динамики популяций. В частности, вектор-функция состояния может описывать плотность (во временном смысле) популяций $n$ видов. Обе независимые переменные имеют размерность времени: $t$ - время, в течение которого рассматривается процесс; $s$ - возраст; $s_{0}=0$. Тогда задача (1)-(6) может рассматриваться как задача управления процессом рождаемости с целью достижения в конечный момент времени $t_{1}$ заданной плотности $z(s)$. (1)-(6) может интерпретироваться и как обратная задача восстановления функционального параметра $u(t)$ в (3), при котором в момент $t=t_{1}$ достигается наблюдаемая плотность популяции $z(s)$. Популяции разных видов могут взаимодействовать между собой. Это взаимодействие определяется правой частью системы (1). В частности, (1) можно рассматривать как обобщение классической модели «хищник - жертва» на случай, учитывающий возрастное распределение особей.

Задача (1)-(6) исследуется при следующих предположениях на ее параметры. 
1. Матричная функция $B(t, s)$ и вектор-функция $r(t, s)$ непрерывны на $T \times S$.

2. Матричная функция $C(u, t)$ и вектор-функция $q(u, t)$ непрерывны на $U \times T$.

3. Функции $x^{0}(s), z(s)$ непрерывны на отрезке $S$.

При сделанных предположениях решение задачи Коши (3), (4) существует и единственно в классе абсолютно непрерывных на $T$ функций. Классического же решения начально-краевой задачи для гиперболической системы (1)-(4), вообще говоря, не существует. Это связано с тем, что для существования и единственности классического решения требуется не только выполнение дополнительных условий, обеспечивающих гладкость $x^{0}(s), x\left(t, s_{0}\right)$, но и выполнение условий согласования более высокого порядка по сравнению с (4). Эти условия неизбежно налагают дополнительные предположения на правые части гиперболической системы [8] и ведут к дополнительным предположениям на управляющие воздействия.

В нашем случае можно гарантировать, что решения $x(t, s)$ начальнокраевой задачи (1)-(4) являются для каждого фиксированного допустимого уравнения непрерывными в прямоугольнике $T \times S$ функциями. При этом каждая компонента $x_{i}(t, s)$ является непрерывно дифференцируемой функцией вдоль семейства характеристик гиперболической системы [9].

В дальнейшем дифференциальный оператор в левой части (1) будем обозначать следующим образом:

$$
\frac{d x}{d t}=\left(\left(\frac{d x_{1}}{d t}\right),\left(\frac{d x_{2}}{d t}\right), \ldots,\left(\frac{d x_{n}}{d t}\right)\right),
$$

понимая под этим оператор дифференцирования вдоль характеристик системы (1).

\section{2 Неклассическая формула приращения целевого функционала}

Задача (1)-(6) является линейно-квадратичной с выпуклой подынтегральной функцией в (6). Однако в данном случае классическое условие оптимальности типа принципа максимума Л. С. Понтрягина не является достаточным условием оптимальности. Причина этого заключается в билинейности правой части (3), то есть в наличии произведения вида $C(u, t) \cdot x$. Поэтому обычно подобные задачи решаются теми же стандартными способами (методы принципа максимума, градиентные методы, дискретизация и т.п.), которые применяются для общих нелинейных задач. При этом основные вычислительные затраты итерационных методов приходятся на неоднократные интегрирования начально-краевых задач для исходной и сопряженной гиперболических систем.

Основной идеей настоящей работы является редукция исходной задачи (1)-(6) к задаче оптимального управления системой обыкновенных диф- 
А. В. Аргучинцев, В. С. Кедрин, М. С. Кедрина. Вариационное условие оптимальности в задаче управления гиперболическими уравнениями ...

ференциальных уравнений, выполненная с помощью нестандартной формулы приращения целевого функционала второго порядка.

Рассмотрим два произвольных допустимых процесса $\{u, x=x(t, s, u)\}$ и $\{\tilde{u}=u+\Delta u, \tilde{x}=x(t, s, \tilde{u})=x(t, s, u)+\Delta x\}$. Тогда приращение целевого функционала (6) можно записать в виде:

$$
\Delta J(u)=J(\tilde{u})-J(u)=\frac{1}{2} \int_{S}\left[\left\|\tilde{x}\left(t_{1}, s\right)-z(s)\right\|^{2}-\left\|x\left(t_{1}, s\right)-z(s)\right\|^{2}\right] d s .
$$

Здесь по-прежнему под нормой понимается обычная евклидова норма в $n$-мерном пространстве.

Представим квадрат каждой из норм в виде скалярного произведения вектора самого на себя и воспользуемся свойством:

$$
\|\tilde{x}\|^{2}-\|x\|^{2}=\langle x+\Delta x, x+\Delta x\rangle-\langle x, x\rangle=\langle\Delta x, \Delta x\rangle+2\langle x, \Delta x\rangle .
$$

Здесь угловыми скобками обозначены скалярные произведения в $n$ мерном евклидовом пространстве.

Далее выполним следующие преобразования. Обозначим $\Pi=T \times S$. Введем соответственно на множествах $T$ и П пока произвольные $n$ мерные вектор-функции $p(t), \psi(t, s), n \times n$-мерную диагональную матричную функцию $\Psi(t, s)$ и $n \times n$-мерную симметричную матричную функцию $\mathrm{P}(t)$.

Добавим в формулу приращения (7) четыре нулевых слагаемых

$$
\begin{gathered}
\int_{T}\left\langle p(t), \frac{\partial \Delta x\left(t, s_{0}\right)}{\partial t}-\Delta_{\tilde{u}} C(u(t), t) \tilde{x}\left(t, s_{0}\right)-C(u(t), t) \Delta x\left(t, s_{0}\right)-\Delta q(u(t), t)\right\rangle d t ; \\
\iint_{\Pi}\left\langle\psi(t, s),\left(\frac{d \Delta x}{d t}\right)-B(t, s) \Delta x(t, s)\right\rangle d t d s ; \\
\int_{T}\left\langle P(t) \Delta x\left(t, s_{0}\right), \frac{\partial \Delta x\left(t, s_{0}\right)}{\partial t}-\Delta_{\tilde{u}} C(u, t) \tilde{x}\left(t, s_{0}\right)-C(u, t) \Delta x\left(t, s_{0}\right)-\Delta q(u(t), t)\right\rangle d t ;
\end{gathered}
$$

$$
\iint_{\Pi}\left\langle\Psi(t, s) \Delta x(t, s),\left(\frac{d \Delta x}{d t}\right)-B(t, s) \Delta x(t, s)\right\rangle d t d s .
$$

Здесь $\Delta_{\tilde{u}} C(u, t)=C(\tilde{u}, t)-C(u, t), \Delta q(\tilde{u}, t)=q(\tilde{u}, t)-q(u, t)$.

Применим формулы интегрирования по частям и введем сопряженные задачи

$$
\begin{aligned}
& \frac{\partial \psi}{\partial t}+\frac{\partial \psi}{\partial s}=-B(t, s) \psi,(t, s) \in \Pi, \\
& \psi\left(t_{1}, s\right)=z(s)-x\left(t_{1}, s\right), s \in S, \\
& \psi\left(t, s_{1}\right)=0, t \in T
\end{aligned}
$$




$$
\begin{aligned}
& \dot{p}=-\psi\left(t, s_{0}\right)-C^{T}(u(t), t) p, t \in T, \\
& p\left(t_{1}\right)=0 ; \\
& \frac{\partial \Psi}{\partial t}+\frac{\partial \Psi}{\partial s}=-2 \Psi B(t, s),(t, s) \in \Pi, \\
& \Psi\left(t_{1}, s\right)=-E, s \in S, \\
& \Psi\left(t, s_{1}\right)=0, t \in T ; \\
& \dot{\mathrm{P}}=-\Psi\left(t, s_{0}\right)-C^{T}(u(t), t) \mathrm{P}-\mathrm{P} C(u(t), t), \\
& \mathrm{P}\left(t_{1}\right)=0 .
\end{aligned}
$$

Под нулями в соответствующих условиях (8) и (9) понимаются $n$ мерные вектора, все компоненты которых равны нулю. Под нулями в (10) и (11) понимаются $n \times n$-матрицы, состоящие из нулевых элементов. $E-$ единичная матрица.

Решения задач Коши для обыкновенных дифференциальных уравнений (9) и (11) в силу сделанных выше предположений являются абсолютно непрерывными на $T$ функциями. В начально-краевых условиях для гиперболических систем (8) и (10) невозможно добиться выполнения условий согласования типа (4). Решения понимаются в указанном выше обобщенном смысле, но можно гарантировать их непрерывность только по отдельности в областях $t<s$ и $t>s$. Прямая $t=s$ задает возможную линию разрыва. Отметим также, что в силу диагональности матрицы $\Psi$ система матричных уравнений в (10) представляет собой систему из $n$ скалярных уравнений. Задача (10) не содержит управляющих функций. Поэтому она может быть решена только один раз.

После всех проделанных преобразований формула приращения (7) примет вид:

$$
\begin{aligned}
& \Delta J(u)=-\int_{T}\left\langle p(t, u)+\mathrm{P}(t, u)\left(x\left(t, s_{0}, \tilde{u}\right)-x\left(t, s_{0}, u\right)\right),\right. \\
& \left.\left.\Delta_{\tilde{u}} C(u(t), t) x\left(t, s_{0}, \tilde{u}\right)+\Delta q(u(t), t)\right)\right\rangle d t .
\end{aligned}
$$

Формула (12) справедлива для любой пары допустимых процессов. Она является точной, то есть не содержит остаточных членов. Но «платой» за это является присутствие в формуле значений вектор-функции состояния, подсчитываемых на новом управлении $\tilde{u}=u+\Delta u$.

\section{3 Вариационное условие оптимальности и его применение}

Полученная в конце предыдущего раздела формула приращения (12) лежит в основе соответствующего необходимого и достаточного условия оптимальности вариационного типа.

Зафиксируем произвольное допустимое управление $u(t)$. Ему соответствуют решения сопряженных задач (9) и (11). Подставим соответствую- 
А. В. Аргучинцев, В. С. Кедрин, М. С. Кедрина. Вариационное условие оптимальности в задаче управления гиперболическими уравнениями ...

щие функции $p(t)$ и $\mathrm{P}(t)$ в формулу (12). Обозначим оставшееся произвольным управление $\tilde{u}$ через $\vartheta$. Из (12) следует основной результат данной работы.

Теорема. Допустимое управление $\vartheta^{*}(t)$ доставляет глобальный минимум в задаче оптимального управления (1)-(6) тогда и только тогда, когда для любого допустимого управления $u(t)$ оно доставляет глобальный минимум в задаче оптимального управления системой обыкновенных дифференциальных уравнений:

$$
\begin{aligned}
& \Delta J(\vartheta)=-\int_{T}\left\langle p(t, u)+\mathrm{P}(t, u)\left(y(t, \vartheta)-x\left(t, s_{0}, u\right)\right),\right. \\
& (C(\vartheta(t), t)-C(u(t), t)) y(t, \vartheta)+q(\vartheta(t), t)-q(u(t), t)\rangle d t \rightarrow \min , \\
& \dot{y}=C(\vartheta(t), t) y+q(\vartheta(t), t), t \in T, \\
& y\left(t_{0}\right)=x^{0}\left(s_{0}\right), \vartheta(t) \in U, t \in T .
\end{aligned}
$$

Сделаем несколько замечаний.

Во-первых, теорема справедлива для любого фиксированного управления $u(t)$. Это связано с тем, что формула приращения (12) верна для любой пары допустимых процессов.

Во-вторых, теорема является необходимым и достаточным условием глобальной оптимальности. Это крайне важно для обратных задач математической физики. Отметим, что принцип максимума Л. С. Понтрягина в данной задаче не является глобальным условием оптимальности, несмотря на выпуклость целевого функционала и почти полную линейность динамической системы.

Практическая ценность результата заключается в том, что на его основе можно провести редукцию исходной задачи (1)-(6) к значительно более простой задаче (13).

Изложим схему редукции. Пусть $u(t)$ - некоторое допустимое управление в задаче (1)-(6). Найдем соответствующее данному управлению состояние $x=x(t, s, u)$. Составим сопряженные задачи (8)-(11). Задача (10) не зависит от управления. Задача (11) решается на основе (10) и известного $u(t)$. Для вычисления функции $p(t, u)$, фигурирующей в (13), нужно знать $\psi\left(t, s_{0}\right)$. Для этого, в свою очередь, нужно решить задачу (8). Но все эти интегрирования гиперболических и обыкновенных дифференциальных уравнений нужно проделать лишь только один раз. В результате приходим к задаче оптимального управления (13), в которой функции $u(t)$, $p(t, u), \mathrm{P}(t, u)$ и $y=x\left(t, s_{0}, u\right)$ известны. Данная задача представляет собой задачу оптимального управления линейной системой обыкновенных дифференциальных уравнений с квадратичным целевым функционалом. Для ее решения можно применять весь арсенал методов оптимального управления обыкновенными дифференциальными уравнениями [10-15]. 
В силу билинейности динамической системы в (13) особенно эффективным представляется использование специализированных методов, предложенных В. А. Срочко [16; 17].

\section{Заключение}

Основным результатом работы является не просто вариационное условие оптимальности, а редукция исходной задачи к значительно более простой задаче оптимального управления обыкновенной динамической системой. Поэтому, в частности, можно говорить, что любое улучшение допустимого управления в задаче (13) приведет к улучшению соответствующего допустимого управления в исходной задаче. Применение формул приращения целевого функционала второго порядка дает возможность улучшать особые неоптимальные управления. По-видимому, предлагаемый подход может быть применен и к другим задачам подобной структуры. Отсутствие локальности в формулах приращения и необходимости оценок приращения состояния процесса относительно параметров, характеризующих малость варьирования управлений, открывает возможности для исследования целых классов гибридных задач оптимального управления, содержащих обыкновенные дифференциальные уравнения и уравнения с частными производными.

\section{Литература}

1. Аргучинцев А. В., Поплевко В. П. Оптимальное управление в задаче химической ректификации // Известия вузов. Математика. 2012. № 8. С. 53-57. Текст: непосредственный.

2. Demidenko N. Optimal Control of Thermal-engineering Processes in Tube Furnaces // Chem. Petrol. Eng. 2006. V. 186, no. 42. P. 128-130.

3. Petukhov A. Modeling of Threshold Effects in Social Systems Based on Nonlinear Dynamics // Cybernetics and Physics. 2019. V. 8, no. 4. P. 277-287.

4. Аргучинцев А. В., Поплевко В. П. Оптимальное управление начальными условиями канонической гиперболической системы первого порядка на основе нестандартных формул приращения // Известия вузов. Математика. 2008. № 1. С. 3-10. Текст: непосредственный.

5. Розоноэр Л. И. Принцип максимума Л. С. Понтрягина в теории оптимальных систем. I // Автоматика и телемеханика. 1959. Т. 20, № 10. С. 1320-1334. Текст: непосредственный.

6. Ризниченко Г. Ю., Рубин А. Б. Математические методы в биологии и экологии. Биофизическая динамика репродуктивных процессов. Москва: Юрайт, 2019. Ч. 1. 210 с. Текст: непосредственный.

7. Габасов Р., Кириллова Ф. М. Особые оптимальные управления. Москва: URSS, 2018. 256 с. Текст: непосредственный.

8. Годунов С. К. Уравнения математической физики. Москва: Наука, 1979. 392 с. Текст: непосредственный.

9. Рождественский Б. Л., Яненко Н. Н. Системы квазилинейных уравнений и их приложения к газовой динамике. Москва: Наука, 1978. 686 с. Текст: непосредственный. 
А. В. Аргучинцев, В. С. Кедрин, М. С. Кедрина. Вариационное условие оптимальности в задаче управления гиперболическими уравнениями ...

10. Васильев О. В. Лекции по методам оптимизации. Иркутск: Изд-во ИГУ, 1994. 340 с. Текст: непосредственный.

11. Срочко В. А. Итерационные методы решения задач оптимального управления. Москва: Физматлит, 2000. 160 с. Текст: непосредственный.

12. Rao A. V. A Survey of Numerical Methods for Optimal Control // Advances in Astronautical Sciences. 2009. V. 135. P. 1-32.

13. Golfetto W. A., Silva Fernandes S. A Review of Gradient Algorithms for Numerical Computation of Optimal Trajectories // J. Aerosp. Technol. Manag. 2012. V. 4. P. 131-143.

14. Булдаев А. С. Методы возмущений в задачах улучшения и оптимизации управляемых систем. Улан-Удэ: Изд-во Бурят. гос. ун-та, 2008. 260 с. Текст: непосредственный.

15. Булдаев А. С. Операторные уравнения и алгоритмы принципа максимума в задачах оптимального управления // Вестник Бурятского государственного университета. Математика, информатика. 2020. № 1. С. 35-53. Текст: непосредственный.

16. Срочко В. А., Антоник В. Г. Условия оптимальности экстремальных управлений для билинейной и квадратичной задач // Известия высших учебных заведений. Математика. 2016. № 5. С. 86-92. Текст: непосредственный.

17. Срочко В. А., Аксенюшкина Е. В. Параметризация некоторых задач управления линейными системами // Известия Иркутского государственного университета. Сер. Математика. 2019. Т. 30. С. 83-98. Текст: непосредственный.

\section{VARIATIONAL OPTIMALITY CONDITION IN THE CONTROL PROBLEM OF HYPERBOLIC EQUATIONS WITH DYNAMIC BOUNDARY CONDITIONS}

Alexander V. Arguchintsev

Dr. Sci. (Phys. and Math.), Prof.,

Irkutsk State University

1 Karl Marx St., Irkutsk, 664003, Russia

arguch@math.isu.ru

Victor S. Kedrin

Cand. Sci. (Technical Sciences), A/Prof., Irkutsk State University

1 Karl Marx St., Irkutsk, 664003, Russia

kedrinvs@isu.ru

Mariya S. Kedrina

Master's student,

Irkutsk State University

1 Karl Marx St., Irkutsk, 664003, Russia

kedrinams@gmail.com 
Abstract. The paper deals with an optimal control problem for a linear system of first-order hyperbolic equations with a quadratic cost functional and boundary conditions determined from controlled bilinear ordinary differential equations. These ordinary differential equations are linear for state functions with controlled coefficients. Such problems arise in the simulation of some processes of chemical technology, social demographics, and population dynamics. General optimal control methods are used, normally, for these problems because of bilinear ordinary differential equations. The problem is reduced to an optimal control problem for a system of ordinary differential equations. The reduction is based on non-classic exact second-order increment formulas for the cost functional. This treatment allows using some efficient optimal control methods for the problem.

Keywords: hybrid systems; hyperbolic equations; non-classic increment formulas; reduction of optimal control problems.

For citation

Arguchintsev A. V., Kedrin V. S., Kedrina M. S. Variational Optimality Condition in the Control Problem of Hyperbolic Equations with Dynamic Boundary Conditions. Bulletin of Buryat State University. Mathematics, Informatics. 2021; 1: 13-23 (In Russ.).

\section{References}

1. Arguchintsev A. V., Poplevko V. P. Optimal control in chemical fractionation problem. Russian Mathematics. 2012. Vol. 56. Pp. 44-47. DOI: 10.3103/S1066369X12080063.

2. Demidenko N. Optimal Control of Thermal-engineering Processes in Tube Furnaces. Chem. Petrol. Eng. 2006. Vol. 186, no. 42. Pp. 128-130.

3. Petukhov A. Modeling of Threshold Effects in Social Systems Based on Nonlinear Dynamics. Cybernetics and Physics. 2019. Vol. 8, no. 4. Pp. 277-287.

4. Arguchintsev A. V., Poplevko V. P. Optimal Control of Initial Conditions in the Canonical Hyperbolic System of the First Order Based on Nonstandard Increment Formulas. Russian Mathematics. 2008. Vol. 52. Pp. 1-7. DOI:10.1007/s11982-0081001-1.

5. Rozonoer L. I. L. S. Pontryagin's Maximum Principle in the Theory of Optimum Systems. Part I. Autom. and Remote Contr. 1959. Vol. 20. Pp. 1288-1302.

6. Riznichenko G. Yu., Rubin A. B. Mathematical Methods in Biology and Ecology. Biophysical Dynamics of Reproductive Processes. Part 1. Moscow: Urait Publ., 2019. $210 \mathrm{p}$.

7. Gabasov R., Kirillova F. M. Singular Optimal Controls. Moscow: URSS Publ., 2018. $256 \mathrm{p}$.

8. Godunov S. K. Equations of Mathematical Physics. Moscow: Nauka Publ., 1979. $392 \mathrm{p}$.

9. Rozhdestvenskii B. L., Yanenko N. N. Systems of Quasilinear Equations and Their Applications to Gas Dynamics. Moscow: Nauka Publ. 1968. 686 p.

10. Vasilyev O. V. Lectures on Optimization Methods. Irkutsk: Irkutsk State Univ. Publ., 1994. $340 \mathrm{p}$.

11. Srochko V. A. Iterative Methods for Solving Optimal Control Problems. Moscow: Fizmatlit Publ., 2000. 160 p.

12. Rao A. V. A Survey of Numerical Methods for Optimal Control. Advances in Astronautical Sciences. 2009. Vol. 135. Pp. 1-32. 
А. В. Аргучинцев, В. С. Кедрин, М. С. Кедрина. Вариационное условие оптимальности в задаче управления гиперболическими уравнениями ...

13. Golfetto W. A., Silva Fernandes S. A Review of Gradient Algorithms for Numerical Computation of Optimal Trajectories. J. Aerosp. Technol. Manag. 2012. Vol. 4. Pp. 131-143.

14. Buldaev A. S. Perturbation Methods in Problem of the Improvement and Optimization of the Controlled Systems. Ulan-Ude: Buryat State Univ. Publ., 2008. 260 p.

15. Buldaev A. S. Operator Equations and Maximum Principle Algorithms in Optimal Control Problems. Bulletin of Buryat State University. Mathematics, Informatics. 2020. No. 1. Pp. 35-53. DOI: 10.18101/2304-5728-2020-1-35-53.

16. Srochko V. A., Antonik V. G. Optimality Conditions for Extremal Controls in Bilinear and Quadratic Problems. Russian Mathematics. 2016. Vol. 60. Pp. 75-80. DOI:10.3103/S1066369X1605008X.

17. Srochko V. A., Aksenyushkina E. V. Parameterization of Some Linear Systems Control Problems. Bulletin of Irkutsk State University. Series Mathematics. 2019. Vol. 30. Pp. 83-98. DOI:10.26516/1997-7670.2019.30.83.

Статья поступила в редакцию 25.02.2021; одобрена после рецензирования 05.03.2021; принята к публикации 10.03.2021.

The article was submitted 25.02.2021; approved after reviewing 05.03.2021; accepted for publication 10.03.2021. 